PROCEEDINGS OF THE

AMERICAN MATHEMATICAL SOCIETY

Volume 129, Number 2, Pages 337-345

S 0002-9939(00)05795-6

Article electronically published on August 28, 2000

\title{
NOTE ON A DIOPHANTINE INEQUALITY IN SEVERAL VARIABLES
}

JEFFREY T. BARTON, HUGH L. MONTGOMERY, AND JEFFREY D. VAALER

(Communicated by Dennis A. Hejhal)

\begin{abstract}
We establish estimates for the number of points that belong to an aligned box in $(\mathbb{R} / \mathbb{Z})^{N}$ in terms of certain exponential sums. These generalize previous results that were known only in case $N=1$.
\end{abstract}

\section{INTRODUCTION}

Let $\boldsymbol{\xi}_{1}, \boldsymbol{\xi}_{2}, \ldots, \boldsymbol{\xi}_{M}$ be a finite set of points in $(\mathbb{R} / \mathbb{Z})^{N}$. A basic problem in Diophantine approximation is to estimate the number of points in this set which belong to an aligned box in $(\mathbb{R} / \mathbb{Z})^{N}$ from knowledge of the exponential sums

$$
\sum_{m=1}^{M} e\left(\boldsymbol{\ell} \cdot \boldsymbol{\xi}_{m}\right)
$$

where $\boldsymbol{\ell}$ is restricted to a finite subset of $\mathbb{Z}^{N}$ and $e(x)=e^{2 \pi i x}$. The Erdös-Turán inequality, as stated in [2], is a result of this sort, but it is generally not useful when the measure of the box is small. In the case of a small box the usual approach is Vinogradov's "method of little glasses", as discussed in [5], pp. 32-34. In the present note we establish inequalities that are generally sharper and easier to use in applications. For $N=1$ this is described in [1], section 2.1, and in [3], section 1.2. Here we obtain the corresponding inequalities for arbitrary $N$.

Let $\mathcal{B}_{1}$ denote the collection of all normalized characteristic functions $\varphi_{u, v}$ : $\mathbb{R} / \mathbb{Z} \rightarrow \mathbb{R}$ defined by

$$
\varphi_{u, v}(x)=\left\{\begin{array}{l}
1 \text { if } u<x-n<v \text { for some } n \in \mathbb{Z}, \\
\frac{1}{2} \text { if } u-x \in \mathbb{Z} \text { or if } v-x \in \mathbb{Z}, \\
0 \text { otherwise, }
\end{array}\right.
$$

where $u<v<u+1$. Then for each positive integer $L$ let $\mathcal{B}_{1}(L) \subseteq \mathcal{B}_{1}$ be the subcollection of functions $(1.1)$ such that $(v-u)(L+1)$ is a positive integer. We write $\mathcal{B}_{N}$ for the collection of functions $\Phi_{\mathbf{u}, \mathbf{v}}:(\mathbb{R} / \mathbb{Z})^{N} \rightarrow \mathbb{R}$ of the form

$$
\Phi_{\mathbf{u}, \mathbf{v}}(\mathbf{x})=\prod_{n=1}^{N} \varphi_{u_{n}, v_{n}}\left(x_{n}\right)
$$

Received by the editors April 15, 1999.

2000 Mathematics Subject Classification. Primary 11J25, 11K60, 11K38.

The first and third authors' research was supported in part by the National Science Foundation (DMS-9622556) and the Texas Advanced Research Project. 
where $\mathbf{u}$ and $\mathbf{v}$ are points in $\mathbb{R}^{N}$ with $u_{n}<v_{n}<u_{n}+1$ in each coordinate. If $\mathbf{L}$ in $\mathbb{Z}^{N}$ has positive coordinates $L_{n}, n=1,2, \ldots, N$, we write $\mathcal{B}_{N}(\mathbf{L}) \subseteq \mathcal{B}_{N}$ for the subcollection of functions (1.2) such that $\left(v_{n}-u_{n}\right)\left(L_{n}+1\right)$ is a positive integer for each $n=1,2, \ldots, N$. Given $\mathbf{L}$ and $\Phi_{\mathbf{u}, \mathbf{v}}$ in $\mathcal{B}_{N}$ it will be convenient to set

$$
\left(v_{n}-u_{n}\right)\left(L_{n}+1\right)=w_{n}, \quad n=1,2, \ldots, N,
$$

so that $0<w_{n}<L_{n}+1$. Thus $\Phi_{\mathbf{u}, \mathbf{v}}$ belongs to $\mathcal{B}_{N}(\mathbf{L})$ if and only if $w_{n} \in$ $\left\{1,2, \ldots, L_{n}\right\}$ for each $n$. Also, we use the lattice point $\mathbf{L}$ to determine the subset $\mathcal{L}=\mathcal{L}(\mathbf{L}) \subseteq \mathbb{Z}^{N}$ defined by

$$
\mathcal{L}=\left\{\boldsymbol{\ell} \in \mathbb{Z}^{N}:\left|\ell_{n}\right| \leq L_{n}, n=1,2, \ldots, N\right\} .
$$

Now a precise form of the problem we consider in this note is as follows. If $\boldsymbol{\xi}_{1}, \boldsymbol{\xi}_{2}, \ldots, \boldsymbol{\xi}_{M}$ is a finite set of points in $(\mathbb{R} / \mathbb{Z})^{N}$, we wish to estimate sums of the type

$$
\sum_{m=1}^{M} \Phi_{\mathbf{u}, \mathbf{v}}\left(\boldsymbol{\xi}_{m}\right)
$$

from knowledge of the exponential sums

$$
\sum_{m=1}^{M} e\left(\boldsymbol{\ell} \cdot \boldsymbol{\xi}_{m}\right)
$$

where $\boldsymbol{\ell}$ is in $\mathcal{L}$. Here we are concerned with the case where the measure

$$
\int_{(\mathbb{R} / \mathbb{Z})^{N}} \Phi_{\mathbf{u}, \mathbf{v}}(\mathbf{x}) d \mathbf{x}=\prod_{n=1}^{N}\left(v_{n}-u_{n}\right)
$$

of the aligned box is small. Thus our main result is a lower bound for the number of points in the box.

Theorem 1. Let $\Phi_{\mathbf{u}, \mathbf{v}}$ belong to $\mathcal{B}_{N}(\mathbf{L})$ with $w_{1}, w_{2}, \ldots, w_{N}$ determined by (1.3). Assume that $\delta>0$ and $\eta>0$ satisfy

$$
\sum_{n=1}^{N} w_{n}^{-1} \leq \delta
$$

and

$$
\sum_{\substack{\ell \in \mathcal{L} \\ \boldsymbol{\ell} \neq \mathbf{0}}}\left|\sum_{m=1}^{M} e\left(\boldsymbol{\ell} \cdot \boldsymbol{\xi}_{m}\right)\right| \leq \eta M
$$

Then we have

$$
M(1-\delta-\eta-\delta \eta) \prod_{n=1}^{N}\left(v_{n}-u_{n}\right) \leq \sum_{m=1}^{M} \Phi_{\mathbf{u}, \mathbf{v}}\left(\boldsymbol{\xi}_{m}\right) .
$$

As an application of Theorem 1 , we obtain a generalization to $(\mathbb{R} / \mathbb{Z})^{N}$ of the inequality given in [1] as Theorem 2.2 and in [4] as Corollary 21. We write $\|x\|$ for the distance from the real number $x$ to the nearest integer. 
Corollary 2. Let $0<\varepsilon_{n} \leq \frac{1}{2}$ and set $L_{n}=\left[N \varepsilon_{n}^{-1}\right]$ for each $n=1,2, \ldots, N$. Assume that

$$
\max _{1 \leq n \leq N} \frac{\left\|\xi_{n m}\right\|}{\varepsilon_{n}} \geq 1
$$

for each point $\boldsymbol{\xi}_{m}$ in $(\mathbb{R} / \mathbb{Z})^{N}, m=1,2, \ldots, M$. Then we have

$$
M \leq 3 \sum_{\substack{\boldsymbol{\ell} \in \mathcal{L} \\ \boldsymbol{\ell} \neq \mathbf{0}}}\left|\sum_{m=1}^{M} e\left(\boldsymbol{\ell} \cdot \boldsymbol{\xi}_{m}\right)\right|
$$

There is an upper bound analogous to (1.7), but this is much easier to prove.

Theorem 3. Let $\mathbf{L}$ in $\mathbb{Z}^{N}$ have positive coordinates, let $\Phi_{\mathbf{u}, \mathbf{v}}$ belong to $\mathcal{B}_{N}$ with $w_{1}, w_{2}, \ldots, w_{N}$ determined by (1.3). Assume that $\delta>0$ and $\eta>0$ satisfy

$$
\prod_{n=1}^{N}\left(1+w_{n}^{-1}\right) \leq(1+\delta)
$$

and

$$
\sum_{\substack{\boldsymbol{\ell} \in \mathcal{L} \\ \boldsymbol{\ell \neq 0}}}\left|\sum_{m=1}^{M} e\left(\boldsymbol{\ell} \cdot \boldsymbol{\xi}_{m}\right)\right| \leq \eta M
$$

Then we have

$$
\sum_{m=1}^{M} \Phi_{\mathbf{u}, \mathbf{v}}\left(\boldsymbol{\xi}_{m}\right) \leq M(1+\delta+\eta+\delta \eta) \prod_{n=1}^{N}\left(v_{n}-u_{n}\right) .
$$

\section{Preliminary lemmas}

As in 4 we define entire functions $H, J$ and $K$ by

$$
\begin{gathered}
H(z)=\left(\frac{\sin \pi z}{\pi}\right)^{2}\left\{\sum_{m=-\infty}^{\infty} \operatorname{sgn}(m)(z-m)^{-2}+2 z^{-1}\right\}, \\
J(z)=\frac{1}{2} H^{\prime}(z), \text { and } K(z)=\left(\frac{\sin \pi z}{\pi z}\right)^{2} .
\end{gathered}
$$

We note that each of these functions is real valued on the real axis and has exponential type $2 \pi$. The functions $J$ and $K$ are integrable on $\mathbb{R}$ and their Fourier transforms

$$
\widehat{J}(t)=\int_{-\infty}^{\infty} J(x) e(-t x) d x \text { and } \widehat{K}(t)=\int_{-\infty}^{\infty} K(x) e(-t x) d x
$$

are continuous functions supported on $[-1,1]$. These Fourier transforms are given explicitly by

$$
\begin{gathered}
\widehat{J}(t)=\pi t(1-|t|) \cot \pi t+|t| \text { if } 0<|t|<1, \\
\widehat{K}(t)=(1-|t|) \text { if } 0 \leq|t| \leq 1, \\
\widehat{J}(0)=1, \text { and } \widehat{J}(t)=\widehat{K}(t)=0 \text { if } 1 \leq|t| .
\end{gathered}
$$

If $L$ is a positive integer we write $J_{L+1}(z)=(L+1) J((L+1) z)$ so that $J_{L+1}(z)$ has exponential type $2 \pi(L+1)$. Then the Fourier transforms $\widehat{J}$ and $\widehat{J}_{L+1}$ are related 
by the identity $\widehat{J}\left((L+1)^{-1} t\right)=\widehat{J}_{L+1}(t)$ for all real $t$. Similar remarks apply to $K$ and $K_{L+1}$.

For each positive integer $L$ we define trigonometric polynomials $j_{L}(x)$ and $k_{L}(x)$ by

$$
j_{L}(x)=\sum_{m=-\infty}^{\infty} J_{L+1}(x+m)=\sum_{\ell=-L}^{L} \widehat{J}_{L+1}(\ell) e(\ell x)
$$

and

$$
k_{L}(x)=\sum_{m=-\infty}^{\infty} K_{L+1}(x+m)=\sum_{\ell=-L}^{L} \widehat{K}_{L+1}(\ell) e(\ell x) .
$$

The identities (2.2) and (2.3) follow from the Poisson summation formula. We also define the periodic function $\psi(x)$ by

$$
\psi(x)=x-[x]-\frac{1}{2} \text { if } x \notin \mathbb{Z} \text {, and } \psi(x)=0 \text { if } x \in \mathbb{Z} .
$$

The trigonometric polynomials

$$
\begin{aligned}
\psi * j_{L}(x) & =\int_{-1 / 2}^{1 / 2} \psi(x-y) j_{L}(y) d y \\
& =\sum_{\substack{\ell=-L \\
\ell \neq 0}}^{L}(-2 \pi i \ell)^{-1} \widehat{J}\left(\frac{\ell}{L+1}\right) e(\ell x)
\end{aligned}
$$

and $k_{L}(x)$ satisfy the basic inequality

$$
\left|\psi(x)-\psi * j_{L}(x)\right| \leq(2 L+2)^{-1} k_{L}(x)
$$

for all $x$ in $\mathbb{R} / \mathbb{Z}$. A proof of (2.4) is given in [3], Chapter 1, and in 4, Theorem 18 . If $u<v<u+1$, then the periodic functions $\varphi_{u, v}(x)$ and $\psi(x)$ are related by the elementary identity

$$
\varphi_{u, v}(x)=(v-u)+\psi(u-x)+\psi(x-v) .
$$

By combining (2.4) and (2.5) we obtain the inequality

$$
\begin{aligned}
& \left|\varphi_{u, v}(x)-\varphi_{u, v} * j_{L}(x)\right| \\
& \quad \leq\left|\psi(u-x)-\psi * j_{L}(u-x)\right|+\left|\psi(x-v)-\psi * j_{L}(x-v)\right| \\
& \quad \leq(2 L+2)^{-1}\left\{k_{L}(u-x)+k_{L}(x-v)\right\}
\end{aligned}
$$

for all $x$ in $\mathbb{R} / \mathbb{Z}$. Alternatively, (2.6) follows directly from [4], Theorem 19.

We now establish some new inequalities.

Lemma 4. Let $\alpha$ and $\beta$ be real numbers such that $\beta-\alpha=M$ is a positive integer. Then

$$
0 \leq H(x-\alpha)+H(\beta-x)
$$

for all real $x$. 
Proof. From (2.1) we have

$$
\begin{aligned}
H(x)+H(1-x)= & \left(\frac{\sin \pi x}{\pi}\right)^{2}\left\{\sum_{m=-\infty}^{\infty} \operatorname{sgn}(m)(x-m)^{-2}+2 x^{-1}\right. \\
& \left.-\sum_{n=-\infty}^{\infty} \operatorname{sgn}(n-1)(x-n)^{-2}+2(1-x)^{-1}\right\} \\
= & \left(\frac{\sin \pi x}{\pi}\right)^{2}\left\{x^{-2}+2(1-x)^{-1}+2 x^{-1}+(1-x)^{-2}\right\} \\
= & \left(\frac{\sin \pi x}{\pi}\right)^{2}\left\{x^{-1}+(1-x)^{-1}\right\}^{2} \\
\geq & 0
\end{aligned}
$$

for all real $x$. As $H$ is an odd function, we conclude that

$$
H(x)+H(M-x)=\sum_{m=0}^{M-1}\{H(x-m)+H(1+m-x)\} \geq 0 .
$$

The lemma follows from (2.8) by replacing $x$ with $x-\alpha$.

Lemma 5. Assume that the periodic function $\varphi_{u, v}(x)$ belongs to $\mathcal{B}_{1}(L)$. Then the trigonometric polynomial

$$
\varphi_{u, v} * j_{L}(x)=\int_{-1 / 2}^{1 / 2} \varphi_{u, v}(x-y) j_{L}(y) d y
$$

satisfies the inequality

$$
0 \leq \varphi_{u, v} * j_{L}(x) \leq 1
$$

for all $x$ in $\mathbb{R} / \mathbb{Z}$.

Proof. Write

$$
\chi_{u, v}(x)=\frac{1}{2}\{\operatorname{sgn}(x-u)+\operatorname{sgn}(v-x)\}
$$

for the normalized characteristic function of the real interval having endpoints $u$ and $v$. As $u<v<u+1$ we have the obvious identity

$$
\varphi_{u, v}(x)=\sum_{m=-\infty}^{\infty} \chi_{u, v}(x+m) .
$$

Next we apply (2.7) with $\alpha=u(L+1), \beta=v(L+1)$, and conclude that

$$
\begin{aligned}
0 & \leq \frac{1}{2}\{H((L+1)(x-u))+H((L+1)(v-x))\} \\
& =\frac{1}{2}(L+1) \int_{u}^{v} H^{\prime}((L+1)(x-y)) d y \\
& =\int_{-\infty}^{\infty} J_{L+1}(x-y) \chi_{u, v}(y) d y
\end{aligned}
$$


for all real $x$. Then we use $(2.2),(2.11),(2.12)$ and the fact that $J_{L+1}$ is integrable, to establish the inequality

$$
\begin{aligned}
0 & \leq \sum_{n=-\infty}^{\infty} \int_{-\infty}^{\infty} J_{L+1}(x+n-y) \chi_{u, v}(y) d y \\
& =\int_{-\infty}^{\infty} j_{L}(x-y) \chi_{u, v}(y) d y \\
& =\sum_{m=-\infty}^{\infty} \int_{m-1 / 2}^{m+1 / 2} j_{L}(x-y) \chi_{u, v}(y) d y \\
& =\int_{-1 / 2}^{1 / 2} j_{L}(x-y)\left\{\sum_{m=-\infty}^{\infty} \chi_{u, v}(y+m)\right\} d y \\
& =\varphi_{u, v} * j_{L}(x) .
\end{aligned}
$$

Now let

$$
\varphi_{v, u+1}(x)=(u+1-v)+\psi(v-x)+\psi(x-u-1)
$$

be the normalized characteristic function of the complimentary interval in $\mathbb{R} / \mathbb{Z}$. Then

$$
\varphi_{u, v} * j_{L}(x)+\varphi_{v, u+1} * j_{L}(x)=\int_{-1 / 2}^{1 / 2} j_{L}(y) d y=1
$$

and we have just proved that

$$
0 \leq \varphi_{v, u+1} * j_{L}(x)
$$

for all $x$ in $\mathbb{R} / \mathbb{Z}$. Therefore (2.14) and (2.15) verify the inequality on the right of (2.10).

Lemma 6. For each integer $n=1,2, \ldots, N$, let $\alpha_{n}, \beta_{n}$ and $\varepsilon_{n}$ be real numbers such that $0 \leq \alpha_{n} \leq 1,0 \leq \beta_{n}, \alpha_{n}-\beta_{n} \leq \varepsilon_{n}$, and either $\varepsilon_{n}=0$ or $\varepsilon_{n}=1$. Then we have

$$
\prod_{n=1}^{N} \alpha_{n}-\sum_{n=1}^{N} \beta_{n} \prod_{\substack{m \neq 1 \\ m \neq n}}^{N} \alpha_{m} \leq \prod_{n=1}^{N} \varepsilon_{n}
$$

Proof. If $\varepsilon_{n}=1$ for each $n=1,2, \ldots, N$, then (2.16) is obvious. Assume that $\varepsilon_{\ell}=0$ for some index $\ell, 1 \leq \ell \leq N$. It follows that $0 \leq \alpha_{\ell} \leq \beta_{\ell}$ and therefore

$$
\prod_{n=1}^{N} \alpha_{n}-\sum_{\substack{n=1 \\ n \neq \ell}}^{N} \beta_{n} \prod_{\substack{m=1 \\ m \neq n}}^{N} \alpha_{m}-\beta_{\ell} \prod_{\substack{m=1 \\ m \neq \ell}}^{N} \alpha_{m} \leq-\sum_{\substack{n=1 \\ n \neq \ell}}^{N} \beta_{n} \prod_{\substack{m=1 \\ m \neq n}}^{N} \alpha_{m} \leq 0 .
$$

This proves the lemma.

Let $\mathbf{L}$ be a point in $\mathbb{Z}^{N}$ with positive coordinates and $\Phi_{\mathbf{u}, \mathbf{v}}$ a function in $\mathcal{B}_{N}$ having the representation (1.2). For each integer $n=1,2, \ldots, N$, we define trigonometric polynomials

$$
\alpha_{n}\left(x_{n}\right)=\varphi_{u_{n}, v_{n}} * j_{L_{n}}\left(x_{n}\right)
$$

and

$$
\beta_{n}\left(x_{n}\right)=\left(2 L_{n}+2\right)^{-1}\left\{k_{L_{n}}\left(x_{n}-u_{n}\right)+k_{L_{n}}\left(x_{n}-v_{n}\right)\right\} .
$$


We assemble these into multiple trigonometric polynomials

$$
\begin{gathered}
A(\mathbf{x})=\prod_{n=1}^{N} \alpha_{n}\left(x_{n}\right), \\
B(\mathbf{x})=\sum_{n=1}^{N} \beta_{n}\left(x_{n}\right) \prod_{\substack{m=1 \\
m \neq n}}^{N} \alpha_{m}\left(x_{m}\right),
\end{gathered}
$$

and

$$
C(\mathbf{x})=\prod_{n=1}^{N}\left\{\alpha_{n}\left(x_{n}\right)+\beta_{n}\left(x_{n}\right)\right\} .
$$

Here $A, B$ and $C$ depend on $\mathbf{u}$ and $\mathbf{v}$, but we drop reference to these points so as to simplify our notation. It is clear that the Fourier coefficients of $A, B$ and $C$ are supported on $\mathcal{L} \subseteq \mathbb{Z}^{N}$. In particular, we find that

$$
\begin{aligned}
\widehat{A}(\mathbf{0}) & =\int_{(\mathbb{R} / \mathbb{Z})^{N}} A(\mathbf{x}) d \mathbf{x}=\prod_{n=1}^{N}\left(v_{n}-u_{n}\right), \\
\widehat{B}(\mathbf{0}) & =\sum_{n=1}^{N}\left(L_{n}+1\right)^{-1} \prod_{\substack{m=1 \\
m \neq n}}^{N}\left(v_{m}-u_{m}\right) \\
& =\left\{\sum_{n=1}^{N} w_{n}^{-1}\right\} \prod_{m=1}^{N}\left(v_{m}-u_{m}\right),
\end{aligned}
$$

and

$$
\begin{aligned}
\widehat{C}(\mathbf{0}) & =\prod_{n=1}^{N}\left\{\left(v_{n}-u_{n}\right)+\left(L_{n}+1\right)^{-1}\right\} \\
& =\left\{\prod_{n=1}^{N}\left(1+w_{n}^{-1}\right)\right\} \prod_{m=1}^{N}\left(v_{m}-u_{m}\right) .
\end{aligned}
$$

In case $A, B$ and $C$ take nonnegative values, we also get the estimates

$$
|\widehat{A}(\ell)| \leq \widehat{A}(\mathbf{0}), \quad|\widehat{B}(\ell)| \leq \widehat{B}(\mathbf{0}) \text { and }|\widehat{C}(\ell)| \leq \widehat{C}(\mathbf{0})
$$

for all $\ell$ in $\mathbb{Z}^{N}$. Lemma 5 shows that $A$ and $B$ take nonnegative values if $\Phi_{\mathbf{u}, \mathbf{v}}$ belongs to $\mathcal{B}_{N}(\mathbf{L})$, while (2.6) implies that $C$ always takes nonnegative values.

Theorem 7. We have

$$
\Phi_{\mathbf{u}, \mathbf{v}}(\mathbf{x}) \leq C(\mathbf{x})
$$

for all $\mathbf{x}$ in $(\mathbb{R} / \mathbb{Z})^{N}$, and if $\Phi_{\mathbf{u}, \mathbf{v}}$ belongs to $\mathcal{B}_{N}(\mathbf{L})$, then

$$
A(\mathbf{x})-B(\mathbf{x}) \leq \Phi_{\mathbf{u}, \mathbf{v}}(\mathbf{x})
$$

for all $\mathbf{x}$ in $(\mathbb{R} / \mathbb{Z})^{N}$.

Proof. The inequality (2.24) is obvious from (2.6) and the definition of $C$.

In order to verify $(2.25)$ let

$$
E_{\mathbf{u}, \mathbf{v}}=\left\{\mathbf{x} \in(\mathbb{R} / \mathbb{Z})^{N}: x_{n}=u_{n} \text { or } x_{n}=v_{n} \text { for some } n, 1 \leq n \leq N\right\} .
$$


Then either $\varphi_{u_{n}, v_{n}}\left(x_{n}\right)=0$ or $\varphi_{u_{n}, v_{n}}\left(x_{n}\right)=1$ for each point $\mathbf{x}$ in $(\mathbb{R} / \mathbb{Z})^{N} \backslash E_{\mathbf{u}, \mathbf{v}}$. From Lemma 5 we know that

$$
0 \leq \alpha_{n}\left(x_{n}\right) \leq 1 \quad \text { and } \quad 0 \leq \beta_{n}\left(x_{n}\right)
$$

for all $x_{n}$ in $\mathbb{R} / \mathbb{Z}$. And (2.6) implies that

$$
\alpha_{n}\left(x_{n}\right)-\beta_{n}\left(x_{n}\right) \leq \varphi_{u_{n}, v_{n}}\left(x_{n}\right)
$$

for all $x_{n}$ in $\mathbb{R} / \mathbb{Z}$. It follows using (2.26), (2.27) and Lemma 6 that

$$
A(\mathbf{x})-B(\mathbf{x}) \leq \Phi_{\mathbf{u}, \mathbf{v}}(\mathbf{x})
$$

for all points $\mathbf{x}$ in $(\mathbb{R} / \mathbb{Z})^{N} \backslash E_{\mathbf{u}, \mathbf{v}}$. As the left hand side of (2.28) is a continuous function of $\mathbf{x}$, we have

$$
A(\mathbf{x})-B(\mathbf{x}) \leq 0 \leq \Phi_{\mathbf{u}, \mathbf{v}}(\mathbf{x})
$$

when $\mathbf{x}$ is in $E_{\mathbf{u}, \mathbf{v}}$.

We note that the entire functions $H$ and $K$ satisfy the basic inequality

$$
|\operatorname{sgn}(x)-H(x)| \leq K(x)
$$

for all real $x$. This is established in [4], Lemma 5. If we use (2.29) in place of (2.4) and apply Lemma 6 , then it is possible to construct an entire function of $N$ complex variables having exponential type and such that its restriction to $\mathbb{R}^{N}$ minorizes the characteristic function of an aligned box in $\mathbb{R}^{N}$. We do not pursue these ideas here as we require only the periodic version of this construction.

\section{Proof of Theorems 1 And 3}

Assume, as in the statement of Theorem 1, that $\Phi_{\mathbf{u}, \mathbf{v}}$ belongs to $\mathcal{B}_{N}(\mathbf{L})$. Then we apply $(2.20),(2.21),(2.23)$ and $(2.25)$. In this way we obtain the inequality

$$
\begin{aligned}
M & \prod_{n=1}^{N}\left(v_{n}-u_{n}\right)-\sum_{m=1}^{M} \Phi_{\mathbf{u}, \mathbf{v}}\left(\boldsymbol{\xi}_{m}\right) \\
& \leq M \prod_{n=1}^{N}\left(v_{n}-u_{n}\right)+\sum_{m=1}^{M}\left\{B\left(\boldsymbol{\xi}_{m}\right)-A\left(\boldsymbol{\xi}_{m}\right)\right\} \\
& =\sum_{\boldsymbol{\ell} \in \mathcal{L}} \widehat{B}(\boldsymbol{\ell}) \sum_{m=1}^{M} e\left(\boldsymbol{\ell} \cdot \boldsymbol{\xi}_{m}\right)-\sum_{\substack{\boldsymbol{\ell} \in \mathcal{L} \\
\boldsymbol{\ell} \neq \mathbf{0}}} \widehat{A}(\boldsymbol{\ell}) \sum_{m=1}^{M} e\left(\boldsymbol{\ell} \cdot \boldsymbol{\xi}_{m}\right) \\
& =M\left\{\sum_{k=1}^{N} w_{k}^{-1}\right\} \prod_{n=1}^{N}\left(v_{n}-u_{n}\right)+\sum_{\substack{\ell \in \mathcal{L} \\
\boldsymbol{\ell} \neq \mathbf{0}}}\{\widehat{B}(\boldsymbol{\ell})-\widehat{A}(\boldsymbol{\ell})\} \sum_{m=1}^{M} e\left(\boldsymbol{\ell} \cdot \boldsymbol{\xi}_{m}\right) \\
& \leq M \delta \prod_{n=1}^{N}\left(v_{n}-u_{n}\right)+\{\widehat{B}(\mathbf{0})+\widehat{A}(\mathbf{0})\} \sum_{\substack{\boldsymbol{\ell} \in \mathcal{L} \\
\boldsymbol{\ell} \neq \mathbf{0}}}^{M}\left|\sum_{m=1}^{M} e\left(\boldsymbol{\ell} \cdot \xi_{m}\right)\right| \\
& \leq M(\delta+\eta+\delta \eta) \prod_{n=1}^{N}\left(v_{n}-u_{n}\right) .
\end{aligned}
$$

The inequality (1.7) plainly follows from (3.1).

The proof of Theorem 3 is essentially the same but uses (2.22), (2.23) and (2.24). 


\section{Proof of Corollary 2}

Select $\mathbf{L}$ in $\mathbb{Z}^{N}$ so that $L_{n}=\left[N \varepsilon_{n}^{-1}\right]$ and note that

$$
2 \leq L_{n} \text { and } \frac{N}{L_{n}+1}<\varepsilon_{n}
$$

for each $n=1,2, \ldots, N$. Then select $\mathbf{u}$ and $\mathbf{v}$ in $\mathbb{R}^{N}$ by setting

$$
u_{n}=-N\left(L_{n}+1\right)^{-1} \text { and } v_{n}=N\left(L_{n}+1\right)^{-1}
$$

for each $n=1,2, \ldots, N$. From (1.8) and (4.1) we conclude that

$$
\Phi_{\mathbf{u}, \mathbf{v}}\left(\boldsymbol{\xi}_{m}\right)=0
$$

for each $m=1,2, \ldots, M$. Now let $0<\delta$ and $0<\eta$ satisfy (1.5) and (1.6) in the statement of Theorem 1. In view of (4.2) and the conclusion (1.7) of Theorem 1, we must have

$$
1-\delta-\eta-\delta \eta \leq 0
$$

As $w_{n}=\left(v_{n}-u_{n}\right)\left(L_{n}+1\right)=2 N$ for each $n=1,2, \ldots, N$, we can take $\delta=1 / 2$.

Then $1 / 3 \leq \eta$ follows immediately from (4.3). This verifies the corollary.

\section{REFERENCES}

1. Baker, R.C., Diophantine Inequalities, London Mathematical Society Monographs (New Series), Vol. 1, Clarendon Press, Oxford, 1986. MR 88f:11021

2. Cochran, T., Trigonometric approximation and uniform distribution modulo one, Proc. American Math. Soc. 103 (1988), 695-702. MR 89j:11071

3. Montgomery, H.L., Ten Lectures on the Interface of Analytic Number Theory and Harmonic Analysis, American Mathematical Society, Providence, RI, 1994. MR 96i:11002

4. Vaaler, J.D., Some extremal functions in Fourier analysis, Bull. American Math. Soc. 12 (1985), 183-216. MR 86g:42005

5. Vinogradov, I.M., The Method of Trigonometrical Sums in the Theory of Numbers, translated by H. Davenport \& K. F. Roth, Interscience, London, 1954. MR 15:941b

Department of Mathematics, Birmingham-Southern College, Birmingham, Alabama 35254

E-mail address: jbarton@bsc.edu

Department of Mathematics, University of Michigan, Ann Arbor, Michigan 48109

E-mail address: hlm@math.lsa.umich.edu

Department of Mathematics, University of Texas, Austin, Texas 78712

E-mail address: vaaler@math.utexas.edu 